\title{
Endoscopic aqueductal stenting in the management of pediatric hydrocephalus
}

\author{
Lelio Guida, MD, ${ }^{1,2}$ Kevin Beccaria, MD, PhD, ${ }^{1}$ Sandro Benichi, MD, ${ }^{1}$ Anaïs Chivet, MD, ${ }^{1}$ \\ Timothée de Saint Denis, MD, ${ }^{1}$ Syril James, MD, ${ }^{1}$ Giovanna Paternoster, MD, ${ }^{1}$ \\ Michel Zerah, MD, PhD, ${ }^{1}$ Stéphanie Puget, MD, PhD, ${ }^{1}$ and Thomas Blauwblomme, MD, PhD ${ }^{1}$
}

1Department of Pediatric Neurosurgery, Hôpital Necker, Assistance Publique Hôpitaux de Paris, Université de Paris, France; and 2Department of Neurosurgery, University of Milan, Italy

OBJECTIVE Pediatric patients with long-term shunts may experience specific complications related to the segregation of the supra- and infratentorial spaces along with different pressure regimens, leading to either mesencephalic syndromes during shunt dysfunction or isolated fourth ventricle (IFV). An accepted treatment to reestablish normal CSF pathways and reequilibrate the transtentorial pressures is endoscopic aqueductal stenting (EAS) to avoid restenosis. In the present paper, the authors studied children treated with EAS during the last decade for both IFV and obstructive hydrocephalus, evaluated its impact on the course of the disease, and identified prognostic factors for EAS success.

METHODS A noninterventional retrospective study of routinely acquired data was performed, including all hydrocephalic children undergoing EAS between 2011 and 2019 at Hôpital Necker, Paris, France. The following variables were analyzed: etiology of hydrocephalus; number of surgeries before and after stent placement; indication for EAS; type of stent connection (i.e., connected or not to a ventriculoperitoneal shunt); and the stent position. Stent failure was defined as the need to perform further shunt revision. Univariate and multivariate analyses were run to identify factors associated with stent failure.

RESULTS Seventeen patients with a mean age at stent placement of 6 years (SD 6.5 years, range 1 month-18 years) and with a mean follow-up after EAS of 47.5 months (SD 33.7 months, range 5-120 months) were included in the analysis. The etiology of hydrocephalus was as follows: obstructive tumoral (41\%), posthemorrhagic (35\%), postinfectious $(12 \%)$, and dysraphism related (12\%). The indication for EAS was IFV (47\%), rostral midbrain dysfunction syndrome $(35 \%)$, prevention of secondary aqueductal stenosis after debulking surgery $(12 \%)$, or primary aqueductal stenosis $(6 \%)$. No transient or permanent neurological deficits related to the procedure were observed. After EAS, 10 patients did not require further surgeries (59\%), and for the others the number of hydrocephalus-related surgeries significantly decreased after stenting. In univariate analysis posthemorrhagic etiology and prevention of aqueductal stenosis were identified as predictors of a good outcome, whereas in multivariate analysis posthemorrhagic hydrocephalus was found to predict a favorable outcome.

CONCLUSIONS The results confirm EAS as a first-line treatment for IFV and suggest its efficacy in changing the history of hydrocephalic patients who have undergone multiple operations and who experience rostral midbrain dysfunction syndrome, as well as efficacy in the prevention of aqueductal stenosis in selected cases of obstructive tumoral hydrocephalus.

https://thejns.org/doi/abs/10.3171/2020.4.PEDS20144

KEYWORDS neuroendoscopy; endoscopic aqueductal stenting; ventriculostomy; ventriculoperitoneal shunt; mesencephalic syndrome; isolated fourth ventricle; hydrocephalus

ABBREVIATIONS EAS = endoscopic aqueductal stenting; ETV = endoscopic third ventriculostomy; IFV = isolated fourth ventricle; IVH = intraventricular hemorrhage; VPS $=$ ventriculoperitoneal shunt.

SUBMITTED February 26, 2020. ACCEPTED April 21, 2020.

INCLUDE WHEN CITING Published online July 3, 2020; DOI: 10.3171/2020.4.PEDS20144. 
$\mathrm{T}$ REATMENT of pediatric aqueductal stenosis was revolutionized in the 1990s by the widespread use of endoscopic third ventriculostomy (ETV). This technique is safe and efficient in purely obstructive forms of hydrocephalus; however, its success depends on the etiology of the disease and decreases dramatically in inflammatory processes (intraventricular hemorrhage $[\mathrm{IVH}]$ or postinfectious hydrocephalus). ${ }^{1,2}$ Randomized trials in neonates have shown that the risk of ETV failure is 1.45 times higher than with ventriculoperitoneal shunts (VPSs), ${ }^{3}$ which are therefore the first-line treatment in this population. However, shunts in obstructive hydrocephalus bring further specific complications; these are related to the segregation of the supra- and infratentorial spaces along with different pressure regimens, leading to either mesencephalic syndromes during shunt dysfunction or isolated fourth ventricle (IFV) when both the inflow and outflow of the fourth ventricle are closed by postinflammatory membranes. ${ }^{1,4}$ Consequently, in rare patients inflammatory etiologies can be responsible for multiple surgeries that are not fully addressed with either shunts or classic ETV. An accepted treatment is then to reestablish normal CSF pathways by the catheterization of the mesencephalic aqueduct with a balloon and placement of a stent to avoid restenosis. ${ }^{5}$ Indications and results of endoscopic aqueductal stenting (EAS) have been reported in fewer than 15 studies, focused on IFV with short follow-ups, and specifically how this procedure could change the course of the disease in terms of decreasing the number of shunt revisions after EAS was not analyzed.

Here, we studied children treated with EAS during the last decade for either IFV or obstructive hydrocephalus, showed a dramatic reduction of the number of shunt-related surgeries, and identified prognostic factors for EAS success.

\section{Methods}

We followed the STROBE statement's guidelines. ${ }^{6}$ As a noninterventional retrospective study of routinely acquired data, written informed consent for this study was waived, and patients were informed they could oppose the use of their health-related data for research purposes.

\section{Study Design and Participants}

This retrospective study included all hydrocephalic children undergoing EAS between 2011 and 2019 at Hôpital Necker, Paris, France, where approximately 1000 hydrocephalus patients per decade are treated. ${ }^{2}$ We excluded from the study patients who underwent stent placement via a microscopic suboccipital approach for IFV. Clinical and imaging data were extracted from the medical charts using the clinical data warehouse at Hôpital Necker. ${ }^{7}$

\section{Variables and Statistical Methods}

The following variables were considered for analysis: etiology of hydrocephalus (posthemorrhagic or postinfectious, dysraphism related, obstructive); number of surgeries before and after stent placement; indication for EAS (IFV, mesencephalic syndrome, prevention of aqueductal stenosis); type of connection (i.e., obstructed stent fixed to

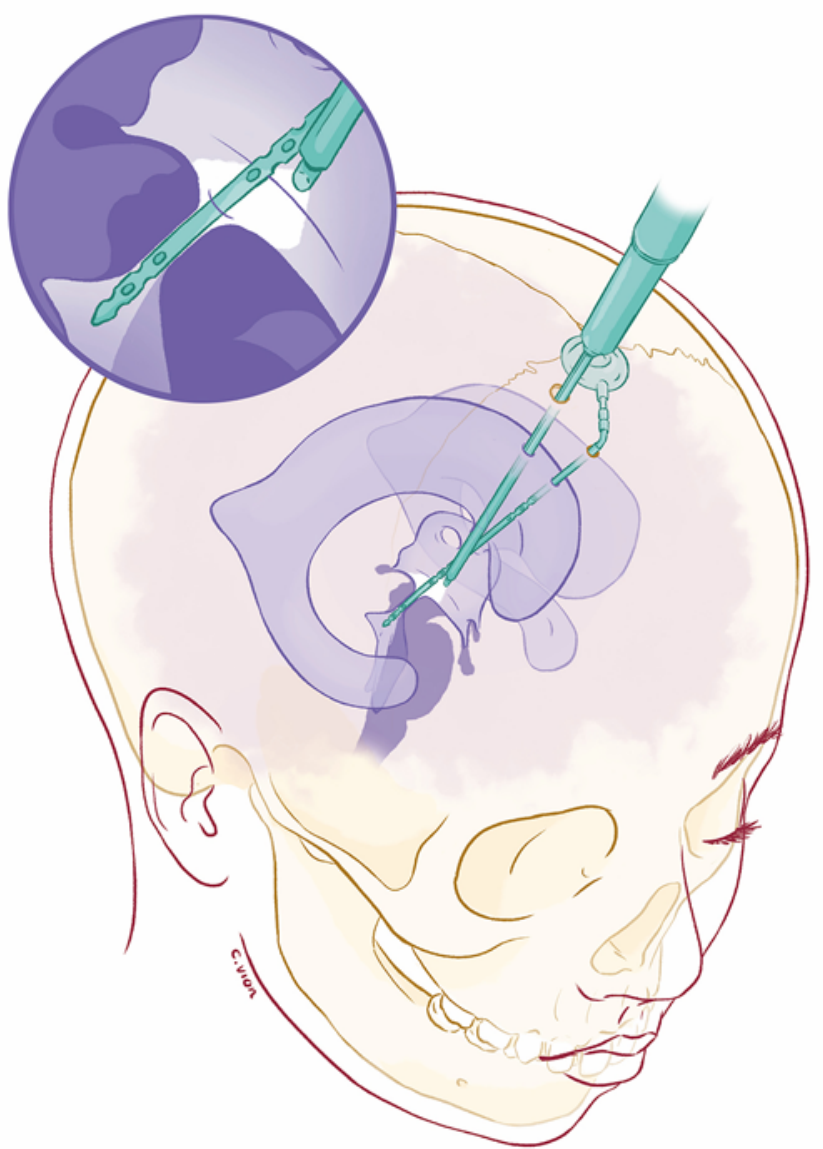

FIG. 1. Artist's depiction of the EAS approach. See text for a step-bystep description of the procedure. Copyright Lelio Guida. Published with permission. Figure is available in color online only.

the epicranium or to an Ommaya reservoir, or stent linked to a VPS); and the stent location. Stent position was defined as optimal for a location in the outlet of the fourth ventricle (below the plane of the inferior colliculi ${ }^{8}$ ), suboptimal if the tip was protruding into the cerebellar parenchyma, and wrong if the stent did not cross the sylvian aqueduct. Stent failure was defined as the need to perform further shunt revision and was studied with the KaplanMeier estimator of the survival function and multivariate Cox proportional hazards regression to determine predictive factors for stent failure. The Wilcoxon signed-rank test was used to compared the number of surgeries before and after EAS within the analyzed subgroups.

Finally, multivariate analysis on outcome was done with binomial logistic regression including all variables with a $\mathrm{p}$ value $<0.1$ in univariate analysis.

\section{Surgical Technique}

Preoperative planning defines the optimal trajectory of both the endoscope and catheter, along with the length of the stent and its perforations in the different ventricles (Fig. 1). Surgery is performed under magnetic neuronavigation guidance (StealthStation, Medtronic). A standard ventricular catheter is prepared by adding more perfora- 
TABLE 1. Summary of the main features of the cohort of 17 pediatric patients with hydrocephalus

\begin{tabular}{|c|c|c|c|c|c|c|c|c|c|c|}
\hline $\begin{array}{l}\text { Case } \\
\text { No. }\end{array}$ & $\begin{array}{l}\text { Age } \\
\text { (mos) }\end{array}$ & Etiology of $\mathrm{HC}$ & $\begin{array}{l}\text { Age at } \\
1 \text { st Op }\end{array}$ & $1 s t O p$ & $\begin{array}{c}\text { No. of } \\
\text { Ops } \\
\text { Prestenting }\end{array}$ & Stent Indication & $\begin{array}{l}\text { Conn to } \\
\text { Shunt } \\
\text { System }\end{array}$ & $\begin{array}{c}\text { Stent } \\
\text { Position }\end{array}$ & $\begin{array}{c}\text { No. of } \\
\text { Ops } \\
\text { Poststenting }\end{array}$ & $\begin{array}{c}\text { FU } \\
\text { (mos) }\end{array}$ \\
\hline 1 & 1 & Postinfectious & 39 days & ETV & 0 & pAS & No & Suboptimal & 3 & 17 \\
\hline 2 & 155 & Supratentorial tumor & $8 \mathrm{yrs}$ & VPS & 3 & Prevention of AS & No & Optimal & 0 & 8 \\
\hline 3 & 39 & Supratentorial tumor & 3 yrs & ETV & 0 & Prevention of AS & No & Suboptimal & 0 & 113 \\
\hline 4 & 43 & Supratentorial tumor & 3 yrs & VPS & 5 & Mesencephalic syndrome & No & Optimal & 0 & 20 \\
\hline 5 & 17 & IVH & 14 days & VPS & 10 & Mesencephalic syndrome & No & Suboptimal & 1 & 5 \\
\hline 6 & 108 & Supratentorial tumor & 9 yrs & ETV & 4 & Mesencephalic syndrome & No & Optimal & 0 & 39 \\
\hline 7 & 220 & Supratentorial tumor & 16 yrs & VPS & 9 & Mesencephalic syndrome & No & Suboptimal & 0 & 36 \\
\hline 8 & 124 & IVH & 29 days & VSGS & 3 & IFV & No & Optimal & 5 & 46 \\
\hline 9 & 17 & IVH & 50 days & VPS & 6 & IFV & No & Optimal & 1 & 120 \\
\hline 10 & 111 & Spinal dysraphism & 54 days & VPS & 5 & IFV & No & Optimal & 3 & 68 \\
\hline 11 & 5 & IVH & 30 days & VSGS & 5 & IFV & No & Optimal & 0 & 70 \\
\hline 12 & 182 & Supratentorial tumor & $14 \mathrm{yrs}$ & VPS & 7 & Mesencephalic syndrome & Yes & Optimal & 0 & 9 \\
\hline 13 & 49 & Supratentorial tumor & $1 \mathrm{yr}$ & VPS & 10 & Mesencephalic syndrome & Yes & Optimal & 0 & 33 \\
\hline 14 & 222 & Postinfectious & 35 days & VPS & 2 & IFV & Yes & Wrong & 3 & 71 \\
\hline 15 & 4 & Spinal dysraphism & 7 days & VSGS & 4 & IFV & Yes & Suboptimal & 3 & 53 \\
\hline 16 & 2 & IVH & 16 days & VSGS & 3 & IFV & Yes & Optimal & 8 & 58 \\
\hline 17 & 4 & IVH & 37 days & VSGS & 3 & IFV & Yes & Optimal & 0 & 42 \\
\hline
\end{tabular}

$\mathrm{AS}=$ aqueductal stenosis; Conn = connection; FU = follow-up; $\mathrm{HC}$ = hydrocephalus; $\mathrm{pAS}$ = primary aqueductal stenosis; VSGS = ventriculosubgaleal shunt.

tions through the use of Mayo scissors or with a bone rongeur. The position of the additional holes is preoperatively planned to allow the drainage of the third ventricle. A 4-cm paramedian linear skin incision allows the surgeon to make 2 burr holes anterior to the coronal suture; the larger and posterior hole is intended for the neuroendoscope and the smaller hole for the ventricular catheter.

The first step is to perform a conventional third ventriculostomy if feasible, and then a spherical isocentric rotation of the endoscope allows visualization of the posterior part of the third ventricle to perform the aqueductoplasty with a double-balloon inflatable probe. The probe is gently and slowly advanced and then inflated while taking care of hemodynamic changes. The ventricular catheter is then inserted under neuronavigation and endoscopic control from the lateral ventricle to the aditus of the aqueduct, where it is gently advanced with the aim of protruding its outlet into the fourth ventricle. To avoid stent migrations and according to the surgeon's indication, the catheter may be either obstructed (i.e., connection of the distal end to a plastic plug and fixation to the pericranium) or connected to an Ommaya reservoir or, rarely, linked to the valve of a VPS.

\section{Results}

\section{Patient Characteristics}

Seventeen patients were included in the analysis (10 boys, 7 girls). The mean age at stent placement was 6 years (SD 6.5 years, range 1 month-18 years), and the patients' characteristics are detailed in Table 1. The etiology of hydrocephalus was as follows: 7 obstructive tumoral ( $41 \%$ of the cases -6 optic pathway glioma; 1 choroid plexus papilloma); 6 posthemorrhagic (35\%); 2 postinfectious (12\%); and 2 dysraphism related (12\%). Fourteen of 17 patients had undergone previous shunt surgery before stent placement (82\% of the cases), 11/14 with the flow-controlled Orbis Sigma 2 (OSV2, Integra Corp.) and 3/14 with the differential pressure Atlas middle pressure valve (Integra Corp.). The mean number of hydrocephalus-related procedures per patient before stenting was 4.65 (SD 3, range $0-10$ ), and in $30 \%$ of patients at least an ETV had been attempted as a rescue procedure in the case of valve dysfunction.

Indications for EAS were as follows: 8 cases of IFV (47\%), 6 rostral midbrain dysfunction syndrome (35\%), 2 prevention of secondary aqueductal stenosis after debulking surgery for third ventricle or hypothalamic tumors $(12 \%)$, and 1 primary aqueductal stenosis (6\%).

\section{Clinical Presentation}

Patients who developed IFV were all symptomatic, with signs of raised intracranial pressure (5 patients, 62.5\%); an isolated Parinaud's sign (2 patients, $25 \%$ ); or cerebellar symptoms (1 patient, $12.5 \%$ ).

Patients with rostral midbrain dysfunction syndrome (sylvian aqueduct syndrome) had an altered state of consciousness (6/6 patients) and midbrain dysfunction signs such as Parinaud's sign (6 patients, 100\%); retraction-convergence nystagmus ( 3 patients, $50 \%$ ); lid retraction and disjunctive eye position (3 patients, 50\%); extrapyramidal abnormal movements (1 patient, 17\%); and pyramidal signs (1 patient, 17\%). All the patients (6/6) had at least one 

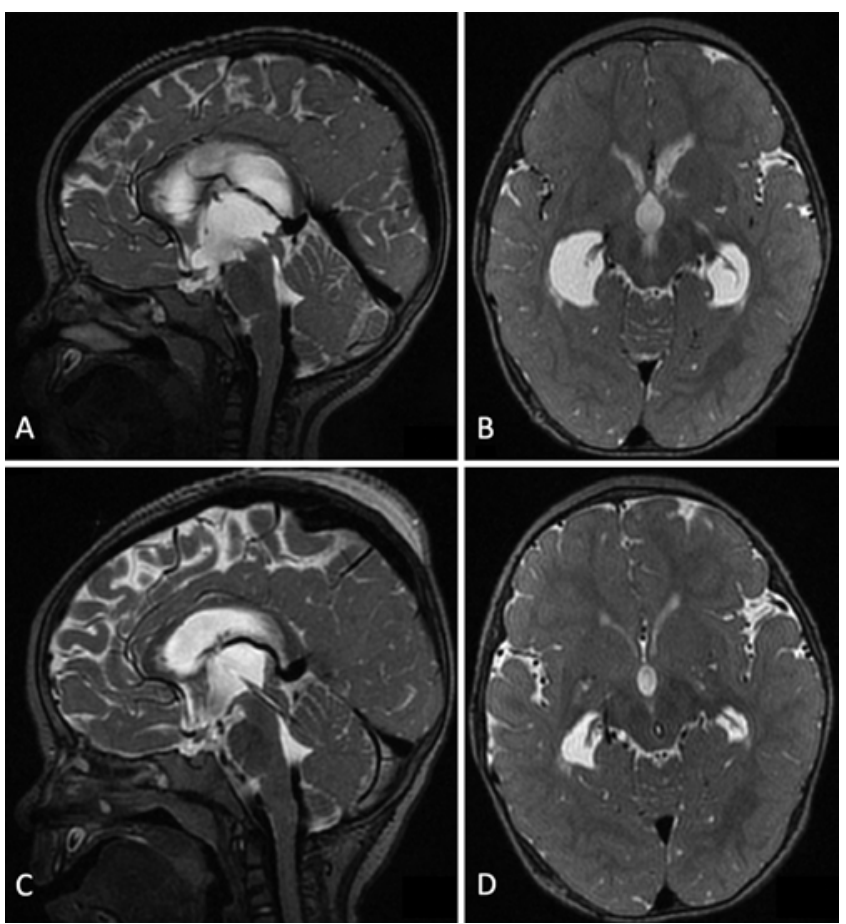

FIG. 2. MRI obtained in a 1-year-old premature child affected by posthemorrhagic hydrocephalus following grade III IVH. The patient was treated using ventriculosubgaleal shunting at birth and then placement of a VPS; the child then experienced a sudden coma and Parinaud's syndrome, with the MRI showing signs of shunt dysfunction. The patient underwent 2 surgeries for shunt revision without clinical improvement in the next 3 days and finally an ETV with restoration of consciousness and disappearance of ocular abnormalities. There were 3 further episodes of global rostral midbrain dysfunction syndrome in the following 5 months, with the MRI showing midbrain hyperintensity in sagittal (A) and axial (B) T2-weighted sequences, ventricular dilatation, and aqueductal stenosis. Given the need to equilibrate the transtentorial pressure gradient, an EAS procedure was performed, with the stent connected to a subcutaneous reservoir and shunt system left in place in the right occipital horn. The patient experienced postoperative regression of midbrain hyperintensity symptoms (C and D) and no further episode at the follow-up.

attempt (range 1-4 attempts) at shunt revision and/or ETV in the days preceding the EAS, with no clinical improvements. In all cases MRI showed a T2 hyperintensity in the periaqueductal region.

Patients who underwent EAS for prevention of aqueductal stenosis had signs and symptoms of raised intracranial pressure (2 patients, $100 \%$ ), whereas the patient with primary aqueductal stenosis had progressive macrocranium and triventricular hydrocephalus.

\section{Surgery}

No transient or permanent neurological deficits related to the procedure were observed. Stent position (Fig. 2) was optimal in 11/17 patients (65\% of the cases); suboptimal in $5 / 17$ (29\%); and wrong, needing replacement in 1 case. In $11 / 17$ procedures $(65 \%)$ the catheter was connected to an Ommaya reservoir or obstructed and sutured to the pericranium, whereas it was directly connected to a shunt sys- tem in 6 cases (35\%). An ETV was performed during the same surgical session in 5 patients (29\%).

\section{Outcome After EAS}

The mean follow-up after EAS was 47.5 months (SD 33.7 months, range 5-120 months). First, aqueductal stenting changed the course of hydrocephalus in this cohort-10 patients did not require further surgeries (59\%), and the Kaplan-Meier estimator showed an overall estimated mean survival time of $49 \pm 13$ months (Fig. 3). Moreover, the number of hydrocephalus-related surgeries significantly decreased after stenting (Wilcoxon test, $\mathrm{Z}=$ $-2.411, p=0.016$ ), from 4.65 to a mean of 1.6 procedures per patient after EAS (SD 2, range 0-8 procedures). Second, we searched for predictive factors of EAS success according to hydrocephalus etiology, stent location, and EAS indication.

In univariate analysis (Fig. 3), comparison of estimated survival time with log-rank test identified posthemorrhagic etiology (log-rank Mantel-Cox, $\chi^{2}=16.688 ; p=0.001$ ) and prevention of aqueductal stenosis (log-rank MantelCox, $\left.\chi^{2}=18.162 ; \mathrm{p}<0.001\right)$ to be predictors of a good outcome. The optimal location of the stent did not reach significance to predict a long survival despite a tendency (log-rank Mantel-Cox, $\left.\chi^{2}=3.671 ; p=0.150\right)$. Reduction of the number of surgeries after EAS was also significant in the following subgroups (Wilcoxon test): patients affected with supratentorial tumors $(Z=-2.201, p=0.028)$; patients developing global rostral midbrain dysfunction syndrome $(Z=-2.207, p=0.027)$; and patients who had the end of the catheter not connected to a VPS system ( $\mathrm{Z}$ $=-2.304, \mathrm{p}=0.021$ ).

In multivariate analysis, hydrocephalus etiology was identified as a factor of EAS success, because patients affected by posthemorrhagic hydrocephalus were 8.2 times less likely to experience stent failure than patients suffering from other kinds of hydrocephalus (hazard ratio 0.122 , $\mathrm{p}=0.08$ ).

\section{Discussion}

In this retrospective single-center study of EAS we show that 1) EAS is safe; 2) EAS is efficient; 3) IFV is not its sole indication; and 4) posthemorrhagic hydrocephalus is predictive of a good outcome.

This study has limitations in regard to its retrospective nature, with a small number of patients treated who had diverse etiologies, limiting the scope of the statistical analysis. However, these drawbacks are explained by the fact that EAS represents a very seldomly performed procedure for the management of hydrocephalus, especially when focusing on the pediatric population. In fact, as summarized in Table 2, only 6 studies with $<65$ patients have been found in the literature analyzing its effectiveness in the pediatric population, and most of them focus on the IFV.

As far as the surgical technique is concerned, we used the precoronal endoscopic approach, which is suitable for children with hydrocephalus and enlarged ventricles as previously described, and we emphasize the utility of neuronavigation for both stent preparation and adequate placement of entry points. ${ }^{4,9-11}$ This is only a slight varia- 


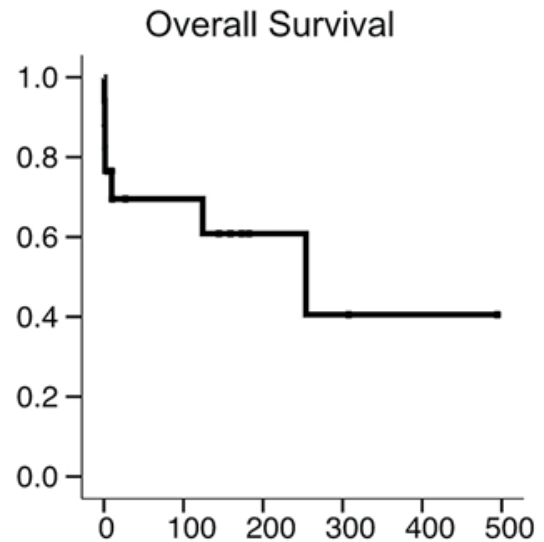

A Time after EAS (weeks)

Number of surgeries

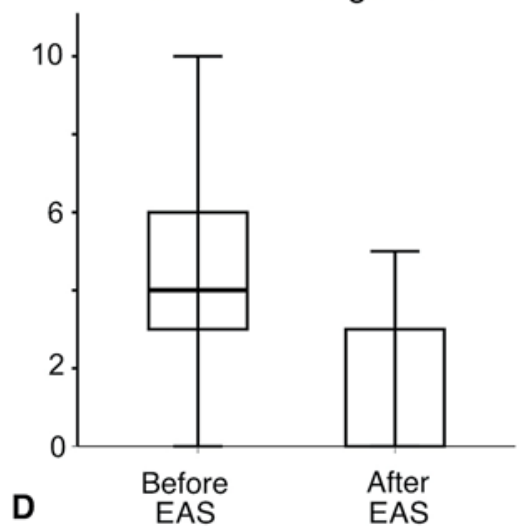

HDC Etiology

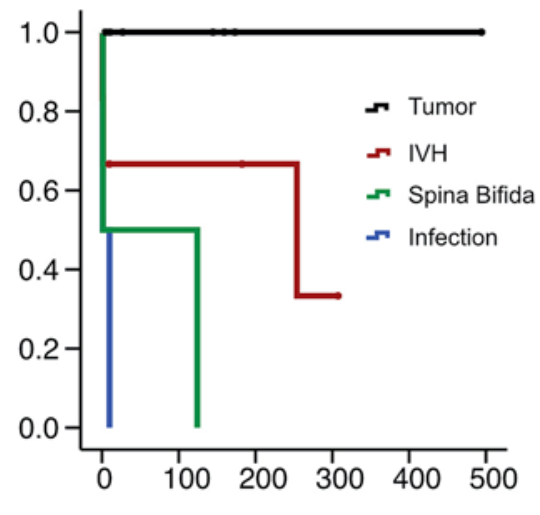

B Time after EAS (weeks)

Number of surgeries

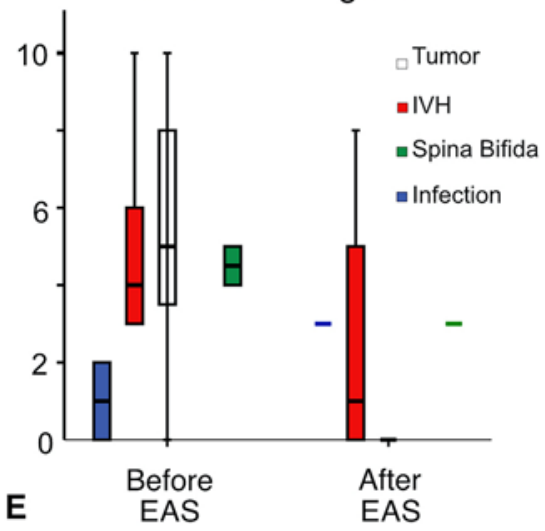

EAS Indication

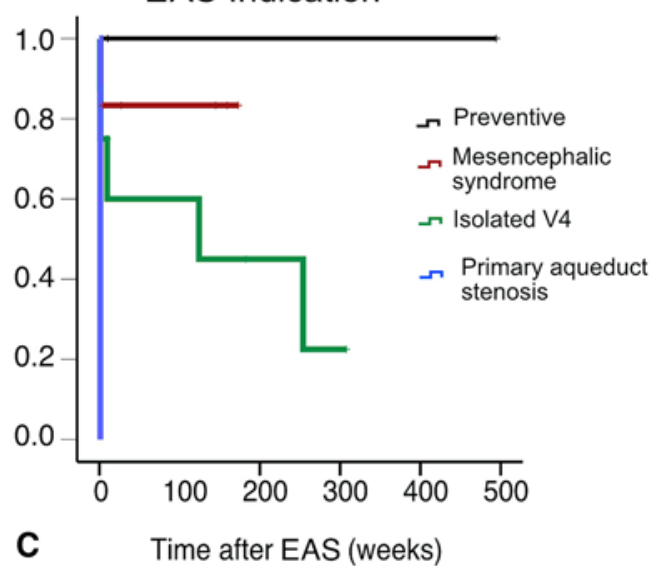

Number of surgeries

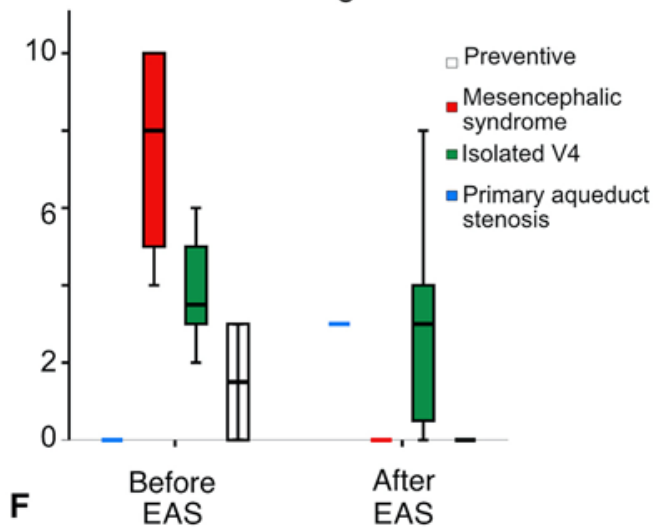

FIG. 3. Summary of the main results. Upper row: Survival plots showing the overall event-free survival curve (A) and the eventfree survival curves according to the etiology of hydrocephalus (B) and the indication for EAS (C). Lower row: Boxplots showing differences in the number of surgeries before and after EAS in the whole cohort (D) and stratified by the etiology of hydrocephalus $(E)$ and the indication for EAS (F). HDC = hydrocephalus; V4 = IFV. Figure is available in color online only.

tion to a standard ETV with a more anterior entry point, but it requires the surgeon to make a second burr hole for the catheter to be coplanar with the mesencephalic aqueduct. This technique is therefore easier with an enlarged foramen of Monro, and this limit could be overcome by the use of flexible endoscopes or with an intracatheter endoscope (such as the Shunt Scope) that allows EAS to be performed through a single trajectory. ${ }^{12,13}$

The indication for EAS in the present study has been broadened and is not limited solely to the treatment of IFVs. Inflammatory processes in the CSF may lead to both obstruction of the outflow through membranous blockage of the aqueduct and/or Magendie foramen, and CSF resorption decrease. Therefore, the endoscopic coronal approach is the treatment of choice for IFV associated with shunt dysfunction or enlarged ventricles because it allows the surgeon to restore the CSF outflow, alleviate brainstem symptoms, and treat hydrocephalus with the connection of the stent to a VPS at the same time. ${ }^{4}$ Excellent results for this approach have also been reported in several pediatric articles. ${ }^{9}, 12,14$

All the same, this mixed mechanism of hydrocephalus can be problematic in mesencephalic syndrome related to shunt dysfunction in patients with aqueductal stenosis. The transtentorial pressure gradient leads to midbrain dysfunction that is ideally treated with third ventriculostomy. ${ }^{1}$ However, postinflammatory adhesions in the subarachnoid spaces can lead to failure of ETV or repeated mesencephalic syndromes, illustrated by the number of redo surgeries (up to 10) in the patients with IVH in our series. Endoscopic stenting could therefore be a secondline option after ETV and shunt revisions in selected cases of posthemorrhagic hydrocephalus. Prevention of aqueductal stenosis and treatment of mesencephalic syndrome in diencephalic tumors are also good indications for EAS in this series and published studies, ${ }^{11}$ because these tumors may not always be amenable to sufficient resection to open the aqueduct or may relapse despite adequate treatment.

\section{Conclusions}

EAS is a rare procedure that has proved to be safe and effective. It is recognized as a first-line procedure in IFVs, but our results suggest that it can also change the history 


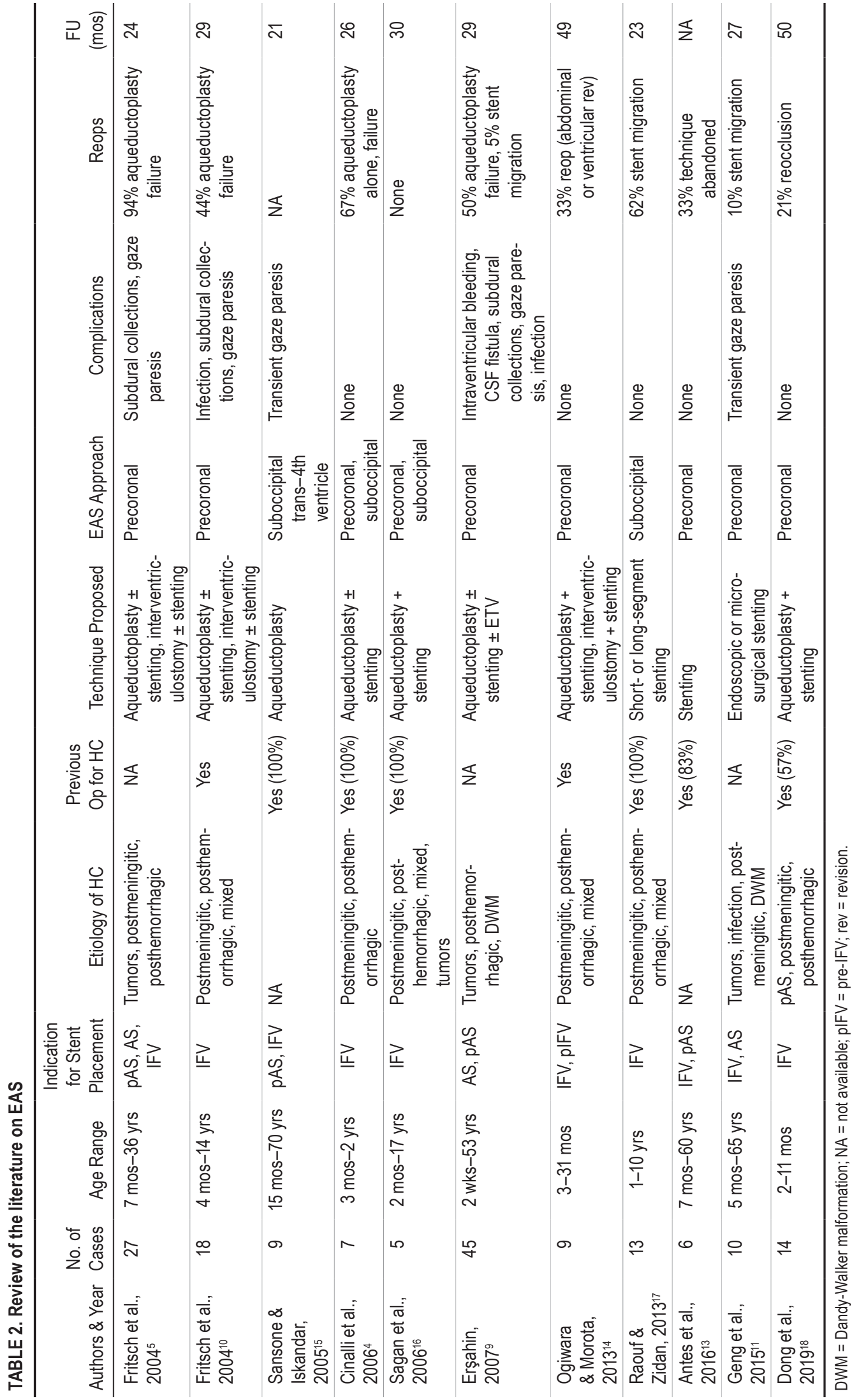


of patients who undergo multiple operations in selected hydrocephalus cases and experience rostral midbrain dysfunction syndrome, as well as be used for children affected by obstructive tumoral hydrocephalus to prevent secondary aqueductal stenosis.

\section{Acknowledgments}

We thank Cassandra Vion for the realization of Fig. 1, and the data science platform of Imagine Institute for data collection.

\section{References}

1. Cinalli G, Sainte-Rose C, Simon I, et al. Sylvian aqueduct syndrome and global rostral midbrain dysfunction associated with shunt malfunction. J Neurosurg. 1999;90(2):227-236.

2. Beuriat P-A, Puget S, Cinalli G, et al. Hydrocephalus treatment in children: long-term outcome in 975 consecutive patients. J Neurosurg Pediatr. 2017;20(1):10-18.

3. Kahle KT, Kulkarni AV, Limbrick DD Jr, Warf BC. Hydrocephalus in children. Lancet. 2016;387(10020):788-799.

4. Cinalli G, Spennato P, Savarese L, et al. Endoscopic aqueductoplasty and placement of a stent in the cerebral aqueduct in the management of isolated fourth ventricle in children. $J$ Neurosurg. 2006;104(1)(suppl):21-27.

5. Fritsch MJ, Kienke S, Mehdorn HM. Endoscopic aqueductoplasty: stent or not to stent? Childs Nerv Syst. 2004;20(3):137-142.

6. von Elm E, Altman DG, Egger M, et al. The Strengthening the Reporting of Observational Studies in Epidemiology (STROBE) statement: guidelines for reporting observational studies. Ann Intern Med. 2007;147(8):573-577.

7. Garcelon N, Neuraz A, Salomon R, et al. A clinician friendly data warehouse oriented toward narrative reports: Dr. Warehouse. J Biomed Inform. 2018;80:52-63.

8. Longatti P, Fiorindi A, Feletti A, et al. Endoscopic anatomy of the fourth ventricle. J Neurosurg. 2008;109(3):530-535.

9. Erşahin Y. Endoscopic aqueductoplasty. Childs Nerv Syst. 2007;23(2):143-150.

10. Fritsch MJ, Kienke S, Manwaring KH, Mehdorn HM. Endoscopic aqueductoplasty and interventriculostomy for the treatment of isolated fourth ventricle in children. Neurosurgery. 2004;55(2):372-379.

11. Geng J, Wu D, Chen X, et al. Aqueduct stent placement: indications, technique, and clinical experience. World Neurosurg. 2015;84(5):1347-1353.
12. Fritsch MJ, Schroeder HWS. Endoscopic aqueductoplasty and stenting. World Neurosurg. 2013;79(2):S20.e15-S20.e18.

13. Antes S, Salah M, Linsler S, et al. Aqueductal stenting with an intra-catheter endoscope-a technical note. Childs Nerv Syst. 2016;32(2):359-363.

14. Ogiwara H, Morota N. Endoscopic transaqueductal or interventricular stent placement for the treatment of isolated fourth ventricle and pre-isolated fourth ventricle. Childs Nerv Syst. 2013;29(8):1299-1303.

15. Sansone JM, Iskandar BJ. Endoscopic cerebral aqueductoplasty: a trans-fourth ventricle approach. J Neurosurg. 2005;103(5)(suppl):388-392.

16. Sagan LM, Kojder I, Poncyljusz W. Endoscopic aqueductal stent placement for the treatment of a trapped fourth ventricle. J Neurosurg. 2006;105(4)(suppl):275-280.

17. Raouf A, Zidan I. Suboccipital endoscopic management of the entrapped fourth ventricle: technical note. Acta Neurochir (Wien). 2013;155(10):1957-1963.

18. Dong X, Zheng J, Xiao Q, et al. Surgical techniques and long-term outcomes of flexible neuroendoscopic aqueductoplasty and stenting in infants with obstructive hydrocephalus: a single-center study. World Neurosurg. 2019;130:98-105.

\section{Disclosures}

The authors report no conflict of interest concerning the materials or methods used in this study or the findings specified in this paper.

\section{Author Contributions}

Conception and design: Blauwblomme, Guida, James, Zerah, Puget. Acquisition of data: Blauwblomme, Guida, Beccaria, Benichi, James, Paternoster. Analysis and interpretation of data: Blauwblomme, Guida. Drafting the article: Blauwblomme, Guida. Approved the final version of the manuscript on behalf of all authors: Blauwblomme. Statistical analysis: Blauwblomme, Guida Administrative/technical/material support: Blauwblomme, Guida. Study supervision: Blauwblomme, Beccaria, Benichi, Chivet, de Saint Denis, James, Paternoster, Zerah, Puget.

\section{Correspondence}

Thomas Blauwblomme: Hôpital Necker Enfants Malades, Paris, France.thomas.blauwblomme@aphp.fr. 